\title{
On the prediction of topology and local properties for optimal trussed structures
}

\author{
J.E. Taylor \\ University of Michigan, Ann Arbor, MI 48109, USA
}

\begin{abstract}
A new formulation is presented for mathematical modelling to predict the distribution of material, material properties, and topology for the optimal design of trussed structures. The design problem is cast in a form to minimize a measure of generalized compliance, which is calculated as a sum over the structure of weighted displacement. Member stiffnesses appear as design variables and, starting with a given ground structure, the solution predicts the optimal layout and distribution of stiffness. The isoperimetric constraint in the reformulated problem measures total cost in generalized form, based on independently specified unit relative cost factors for each truss element. One or another form of optimal design is generated via a process where designated elements in the unit relative cost field are adjusted systematically at each cycle. The generalized cost feature provides as well for the introduction of certain technical constraints into the design problem, e.g. the facility to design around obstacles. Results for each cycle of an algorithm for computational treatment are identified as the solution to a properly posed optimization problem. Computational procedures are demonstrated by the prediction of optimal designs for a variety of truss problems in $2 \mathrm{D}$.
\end{abstract}

\section{Introduction}

The object of this paper is to demonstrate a somewhat generalized formulation for problems in the optimal design of trusses. The analytical model for this formulation is stated as a constrained, nonlinear extremum problem, one which differs from familiar statements for the truss design problem mainly by the form of the argument in its isoperimetric constraint. This argument has total cost evaluated in terms of an independently specified vector of member unit relative cost factors. As would be expected, the consequence for optimal design solutions of change in the unit relative cost field is a first-order effect and, given sufficient insight into the interdependence between unit cost and design, this feature in the model may be exploited to advantage. In addition to the implementation of generalized cost in the model, the design objective is expressed in a form representing generalized compliance, i.e. the objective is evaluated as the sum over the truss of independently weighted nodal displacements (the usual minimum compliance objective is imbedded within this model as the case where the specified unit weights are taken to be proportional to the nodal loads). The generalized compliance feature leads naturally to a non-selfadjoint design problem, and a variational formulation is introduced that proves to be convenient for working with such problems.

The design space for trussed structures is defined in terms of designated sets of support nodes and of interior nodes, and

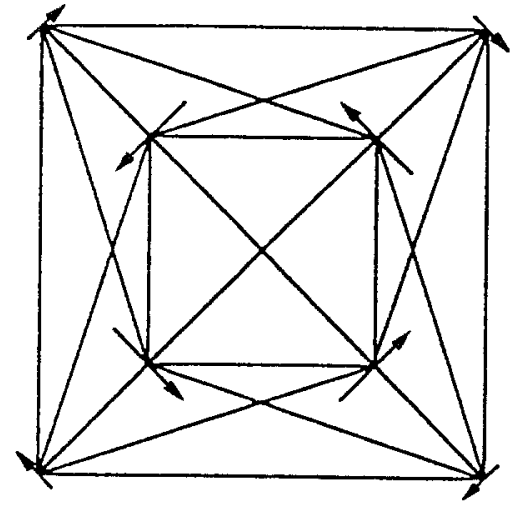

(a)

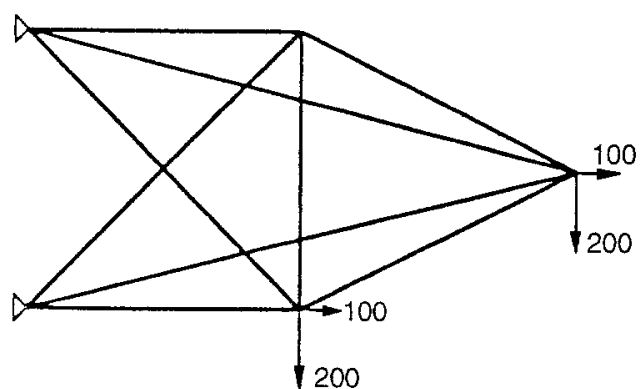

(b)

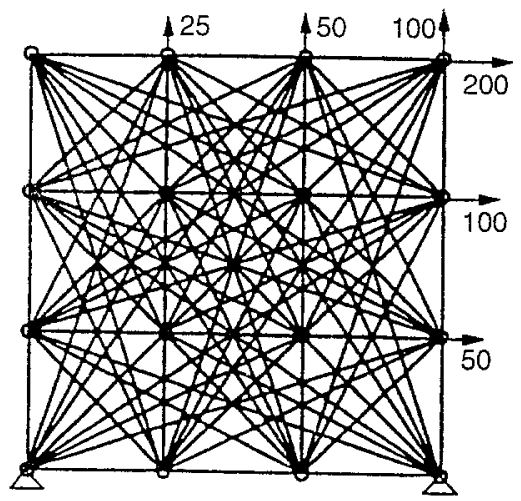

(c)

Fig. 1. Ground structures for three example problems:(a) torque structure, (b) nine-bar truss, (c) four-by-four truss

a connectivity matrix which delineates which pairs of nodes may be connected by a member. The resulting system is 


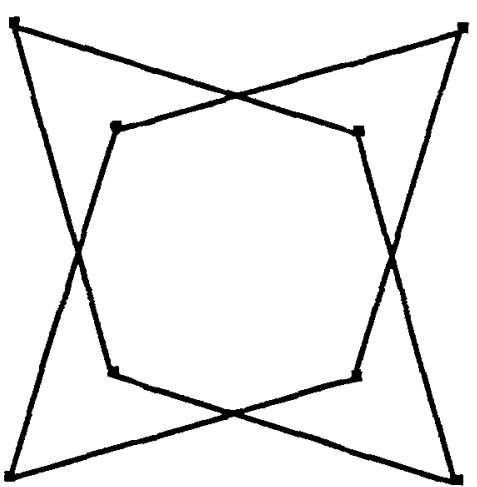

(a)

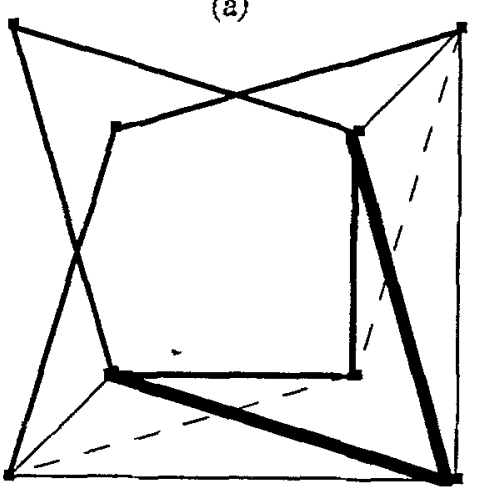

(c)

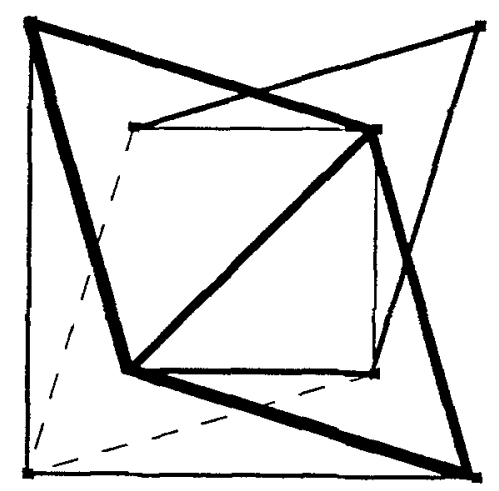

(e)

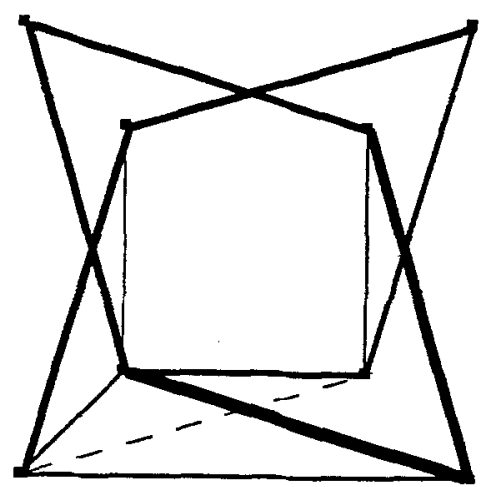

(b)

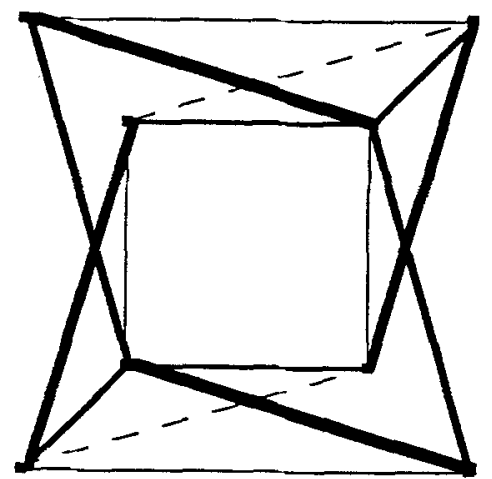

(d)

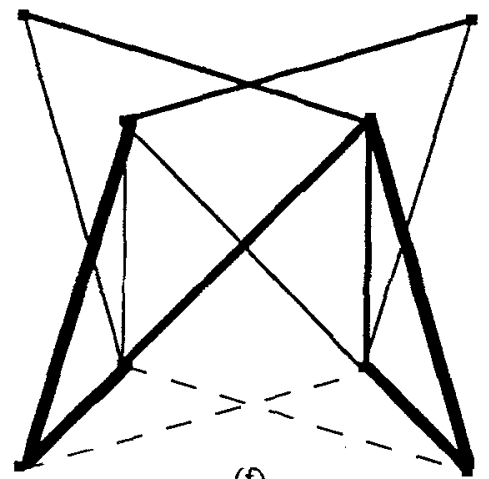

(f)

Fig. 2. Minimum compliance designs for the torque structure: (a) for uniform unit cost, and (b)-(f) for heavily weighted cost on the members shown as dashed lines

termed a ground structure or a structural universe, and it is implicit that the ground structure provides adequately for support of the designated loads. In the present characterization of the design problem, the stiffness properties (crosssectional area $x$ modulus) of the truss members are the design variables. In other words, member material properties and material distribution are lumped in the measure of member stiffness. Thus a solution for the prediction of optimal truss design is comprised of the optimal distribution of member stiffnesses over the prescribed ground structure, and in the case where unit relative member cost is specified to be uniform this result is identical to what would be obtained via conventional models for the problem. On the other hand, it is possible with the appropriate application of the generalized unit relative cost model to obtain special results in truss design. As an example, a refined optimal truss topology or layout can be predicted as the result of a process where the problem is re-solved a number of times with systematic adjustment of the cost factors at each cycle. The model for the design problem used in the cyclic steps of this procedure is otherwise identical to that of the original problem. As a second example, optimal design in the situation where an obstacle or obstacles are present within the design space may be treated by similar means. Programs for the computational treatment of such design problems are established and results are presented for several example design problems of the kinds described.

The developments in this paper are described specifically for linear elastostatic response. The formulation can be extended easily to account for finite deformation mechanics, however, and the steps required to do so are indicated. Also, the techniques applied here for the design of truss topology 


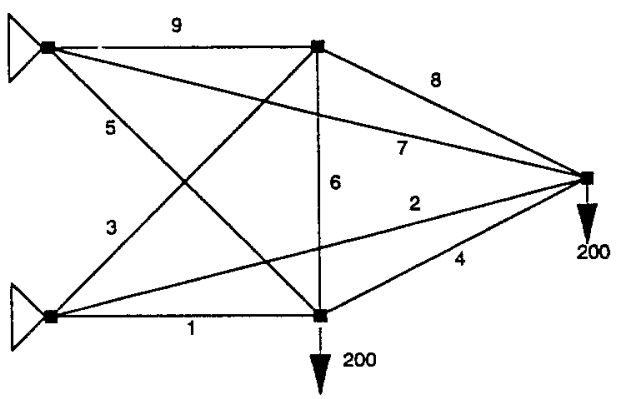

(a)

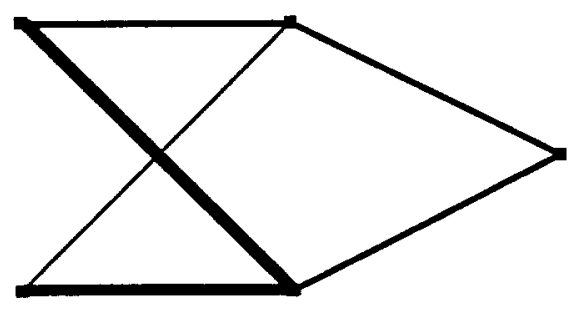

(d)

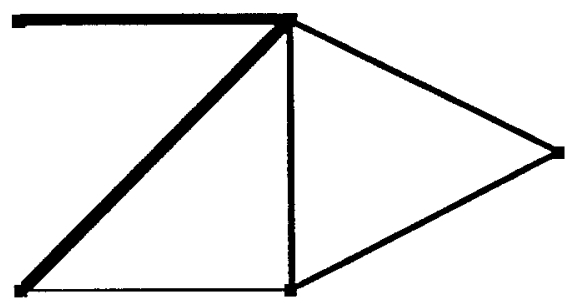

(f)

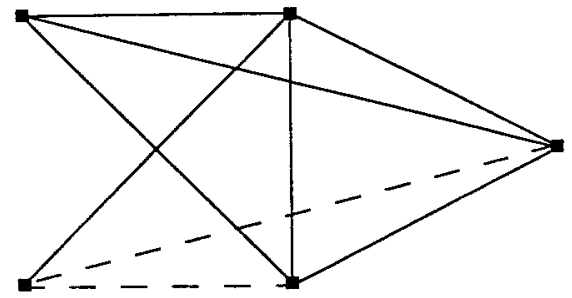

(b)

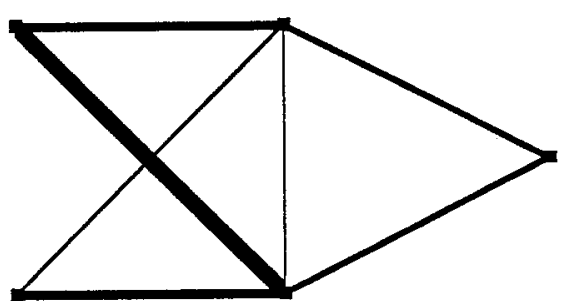

(e)

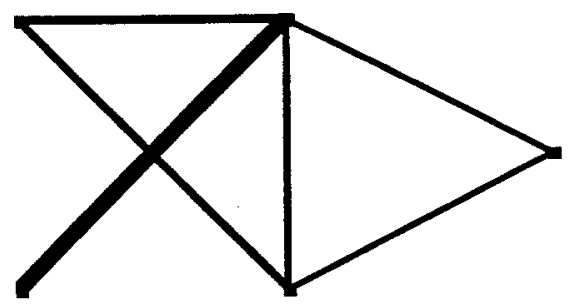

(g)

Fig. 3. Minimum compliance designs for the nine-bar truss: (a) ground structure and loading, (b) unit cost factor varied on members shown as dashed lines, (c) result for uniform cost, (d) weighted cost members at cost factor between 1.25 and 2.25 , (e) weighted cost members at cost factor between 2.5 and 4.1, (f) weighted cost members at cost factor between 4.15 and 4.4

are potentially more widely useful, and it is of interest to note how the introduction of generalized objective and cost in models for the design of continuum structures may be effective for the prediction of topology in that setting. Such considerations, along with elements of the relation between the discrete and continuum design problems, are discussed in the summary.

\section{Formulation for analysis and design}

As indicated above, in the present setting for the design problem the ground structure layout is prescribed, and the truss system design is represented by a vector of memberstiffnesses, say $S_{m}$, lying within the ground structure. Connectivity of the truss can lie anywhere between the full measure associated with the given set of nodes and, at the other limit, the minimum number of members which define a viable structure. The problem can be stated as "predict the design that minimizes a generalized measure of compliance, from among designs that equilibrate the prescribed loads and meet local constraints on $S_{m}$ and a limit on a specific measure of total cost". In this latter (isoperimetric) constraint, total cost is evaluated using a designated "relative cost per unit stiffness" ascribed to each member of the truss. Once again, having total cost represented in this way provides for the study of how optimal layout and material distribution in the truss vary with the (relative) distribution of member cost.

For the present version of minimum generalized compliance design, the objective is to minimize " $Q$-weighted displacements $u$ summed over the truss nodes", i.e. $\sum_{\gamma} \mathbf{Q}_{\gamma}$ $u_{\gamma}$ l, where the (bounded) vector $\mathrm{Q}$ is specified independently of the actual loads. An alternative formulation was presented by Rozvany ( 1989 for an analytical treatment and e.g. Zhou and Rozvany 1992/1993 for the numerical algorithms); there the weighted displacements are constrained to similar effect. System stiffness $K_{\alpha \beta}$ is supposed to be explicit and linear in terms of the member properties $S_{j}$. With the $S_{j}$ as design variables, and omitting at this stage the consideration of generalized local cost, the problem can be described in the more or less typical form

$$
\min _{\left(u_{\gamma}, \nu_{\gamma}, S_{j}\right)} \sum_{\gamma} Q_{\gamma}\left|u_{\gamma}\right|
$$

subject to

$P_{\beta}-\sum_{\alpha} K_{\alpha \beta}\left(S_{j}\right) u_{\alpha}=0, \quad \beta=1,2, \ldots, N$ 


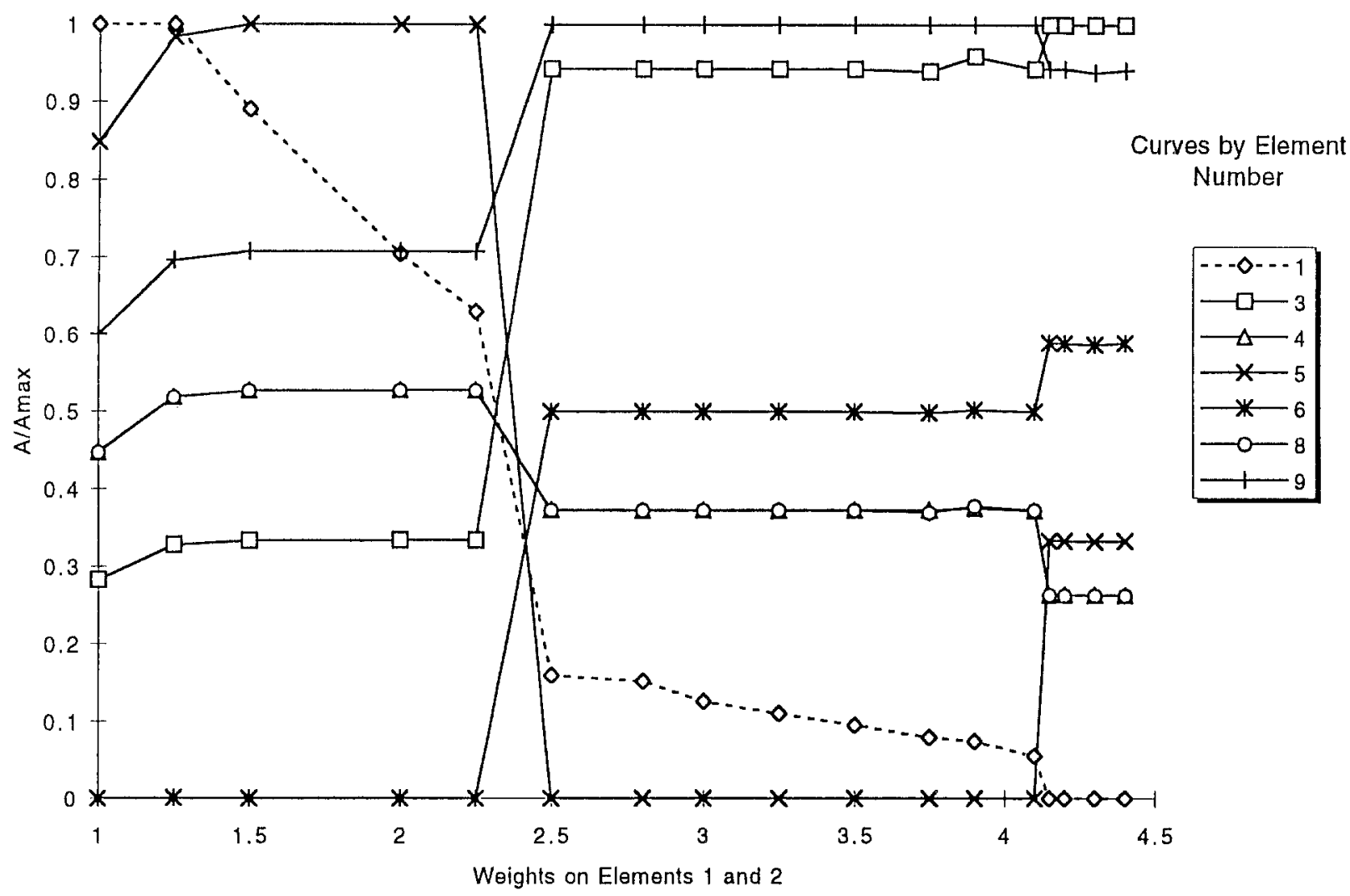

Fig. 4. Evolution of optimal design with increasing weight, for the results shown in Fig. 3

$\ell_{j}\left(\underline{S}_{j}-S_{j}\right) \leq 0, \quad j=1,2, \ldots, M$,

$\sum_{j} S_{j} \ell_{j}-R \leq 0$

Here weights $Q_{\gamma}>0$, nodal loads $P_{\gamma}$, lengths $\ell_{j}$, and bounds $\underline{S}_{j}$ and $R>0$ are data for the combined analysis and design problem; $N$ and $M$ count, respectively, the number of displacement degress of freedom, and the number of members. The first constraint enforces that solution $u_{\gamma}$ equilibrates loads $P_{\gamma}$. This formulation represents truss optimal design within given ground structure that is essentially equivalent to one appearing frequently in earlier works on the subject, with the exception of the form of the objective [truss design is treated at length in the survey article by Rozvany et al. (1995), in the book by Bendsøe (1995), and in papers in the book edited by Bendsøe and Mota Soares (1993), and much of the relatively contemporary literature on the subject is identified in these resources. The papers of Prager (1977), and Taylor and Rossow (1977) may be of historical interest. Models for the treatment of truss design with nonlinear materials are presented by Taylor and Washabaugh (1994)]. The Lagrange multiplier on the equilibrium constraint has the role of adjoint displacement in this formulation, and the condition for stationarity in [T] w.r.t. $u_{\gamma}$ has the form of an adjoint equilibrium equation with loads $Q_{\gamma}$ sgn $u_{\gamma}$. The customary (selfadjoint) form of the minimum compliance problem is recovered from the above statement in the case $Q_{\gamma}$ sgn $u_{\gamma}=P_{\gamma}$.

Essentially the same design problem is to be restated in alternate form, where the structure of the problem is modified so that requirements on deformation kinematics (compatibility conditions) appear explicitly as constraints [Washizu (1982) follows a similar approach, in a different problem setting]. As a result, both member elongations and adjoint elongations, and associated displacements $u_{\gamma}$ and $\nu_{\gamma}$ appear explicitly. The scope of the problem is enlarged as well with the introduction of the measure of generalized local cost, which has the argument of the isoperimetric constraint evaluated using specified unit relative cost factors (weights) $\omega_{j}$. Note that, with the exception of this latter feature, the problem formulated as follows is equivalent to the previous problem $[\mathrm{T}]$ :

$\min _{\left(u_{\gamma}, \nu_{\gamma}, S_{j}, e_{j}, \hat{e}_{j}\right)} \sum_{\gamma} Q_{\gamma}\left|u_{\gamma}\right|$,

subject to

$\left[\sum_{\gamma} P_{\gamma} \nu_{\gamma}-\sum_{j} \frac{S_{j}}{\ell_{j}} e_{j} \hat{e}_{j}\right] \leq 0$,

$\hat{e}_{j}-\sum_{\gamma} \mathbf{B}_{\gamma j} \nu_{\gamma}=0$

$\left.\begin{array}{l}e_{j}-\sum_{\gamma}^{\gamma} \mathbf{B}_{\gamma j} u_{\gamma}=0 \\ \ell_{j}\left(\underline{S}_{j}-S_{j}\right) \leq 0\end{array}\right\} j=1,2, \ldots, M$,

$\sum_{j} \omega_{j} S_{j} \ell_{j}-R \leq 0, \quad \sum_{j} \omega_{j}=M$

Matrix $\mathbf{B}_{\gamma j}$ enforcing compatibility between system and element deformations is determined according to the designated truss layout. Multipliers associated with the elongation/displacement equations are identified with member 


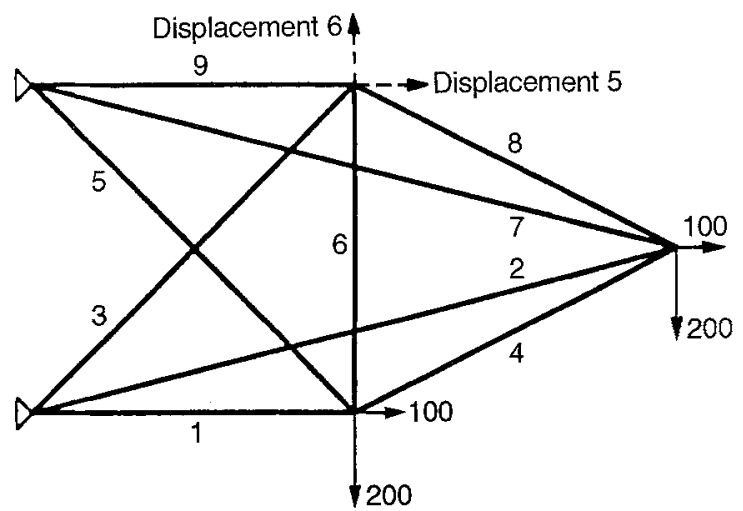

(a)

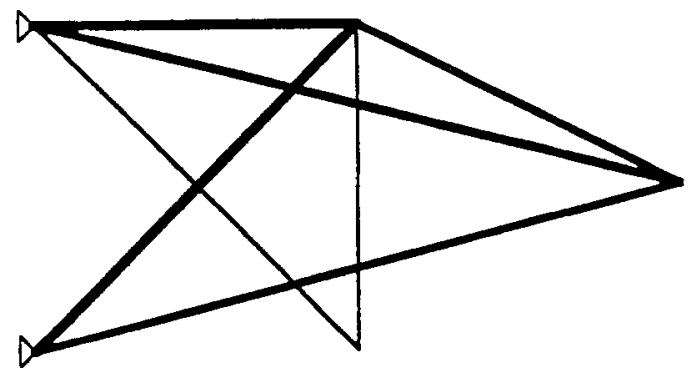

(c)

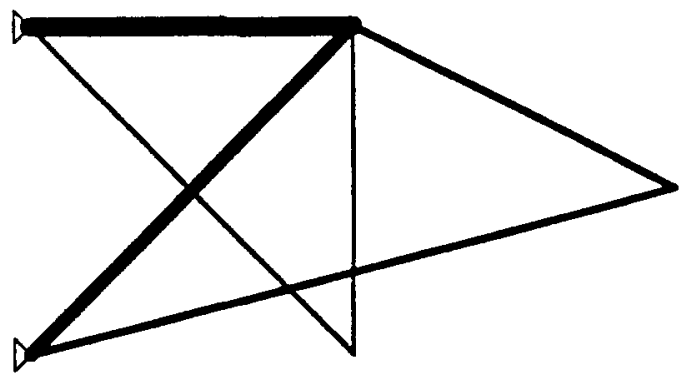

(b)

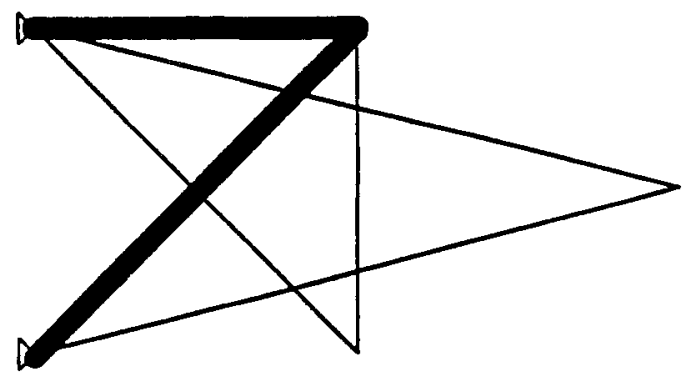

(d)

Fig. 5. Generalized compliance designs for the nine-bar truss: (a) loads and indication of independently weighted displacement components 5 and 6, (b) result for uniformly weighted displacement, (c) and (d) results for weight factors on displacements 5 and 6 equal to 3 and 412 , respectively

forces in the primary and adjoint equilibrium systems, as is to be demonstrated. Modelling the design problem in this alternative form obviates the need to evaluate system stiffness in terms of the member properties. Primary and adjoint equilibrium equations are identified among "the necessary conditions" for problem [Q]; the formulation admits interpretation as a form of generalized complementary potential energy principle [elements of such variational formulations, including nonlinear applications are described by Taylor (1996)].

Representation here of "cost" in terms of unit relative cost factors is the counterpart for discrete structures of the modelling described by Taylor and Washabaugh (1995) for generalized cost in the design of continuum structures. As a final note, one may observe that the problem structure is symmetric in form with respect to $u$ and $\nu$, i.e. the same problem results when the two variables are interchanged.

The isoperimetric constraint in the above formulations is interpreted to express a limit on global cost and accordingly the weights $\omega_{j}$ measure unit relative cost. The following properties associated with the problem statement are noted.

(1) The problem represented is a "properly posed" min problem, i.e. there exists a unique minimizer for [Q].

(2) The set of solutions associated with a sufficiently broad set of values $\omega_{j}$ and with all other data values held fixed, spans the space of all designs admissible for the given layout and within the remaining specified data values.

(3) To represent the converse of (2), for any design within an appropriately normalized set and satisfying the lower bounds on $S_{j}$, there exists an $\omega_{j} ; j=1,2, \ldots, M$ such that the the given design and its associated response are optimal.

Details related to these points are to be discussed on the basis of an interpretation of the necessary conditions for formulation [Q]. The full set of necessary conditions is to be examined next.

Notation $\Lambda, \eta_{j}, \varsigma_{j}, \lambda_{j}$ and $\Gamma$ is introduced to symbolize Lagrange multipliers associated, in order, with the constraints of problem [Q]. Stationarity in [Q] with respect to $u_{\rho}, \nu_{\rho}, e_{k}$ and $\hat{e}_{k}$ requires that the following equations are satisfied at the solution point:

$Q_{\rho}\left(\operatorname{sgn} u_{\rho}\right)-\sum_{j} B_{\rho j} \varsigma_{j}=0$,

$\Lambda P_{\rho}-\sum_{j} B_{\rho j} \eta_{j}=0$

$-\Lambda \frac{S_{k} \hat{e}_{k}}{l_{k}}+\varsigma_{k}=0$

$-\Lambda \frac{S_{k} e_{k}}{l_{k}}+\eta_{k}=0$

From (4) the quantity $\eta_{j}$ represents member force associated with elongation $e_{k}$, and the elimination of $\eta_{j}$, between (2) and (4) leads to system equilibrium equations

$P_{\rho}-\sum_{k} B_{k \rho} \frac{S_{k} e_{k}}{\ell_{\ell}}=0, \quad \rho=1,2, \ldots, N$. 


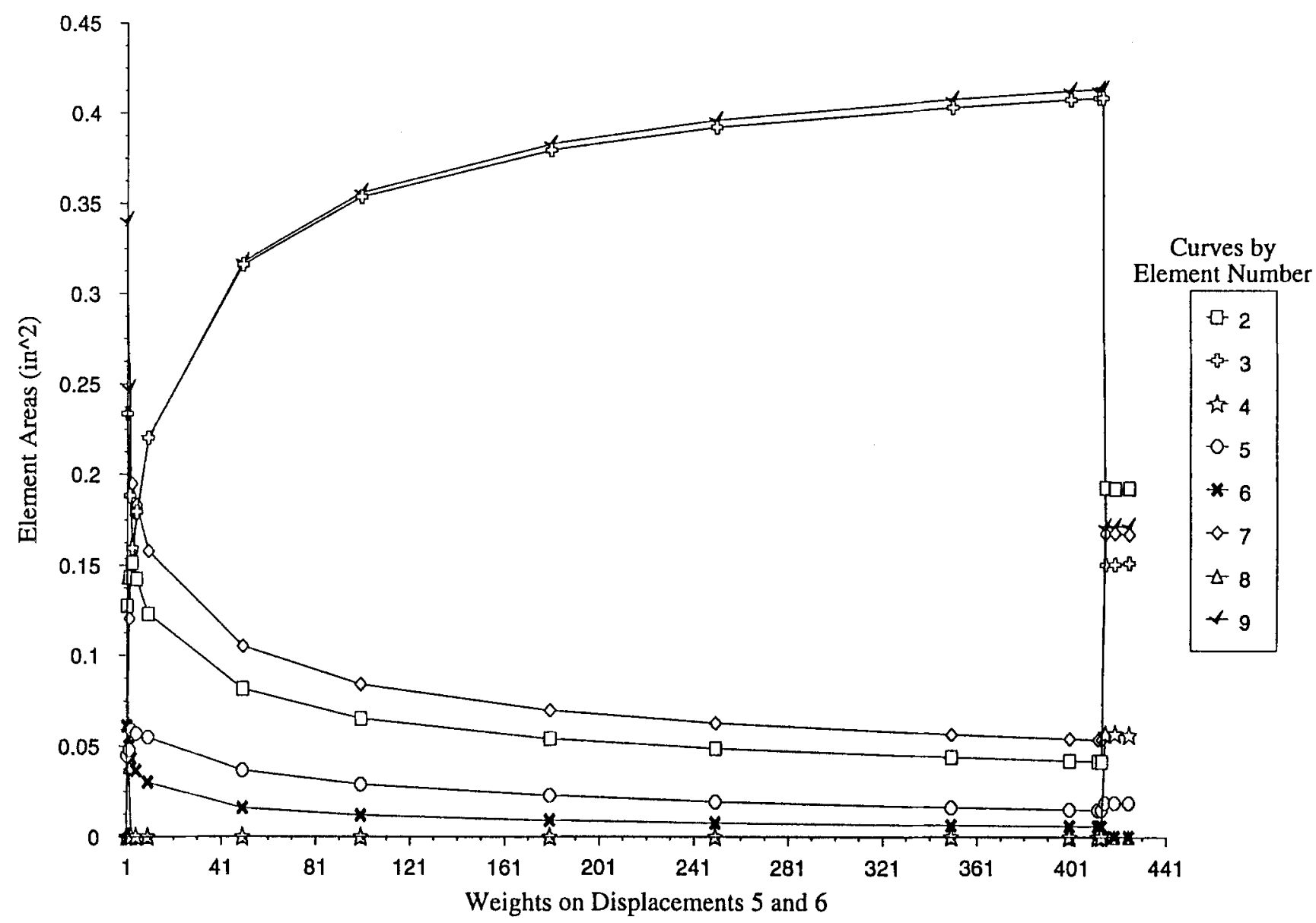

Fig. 6. Evolution of optimal design with increasing weight on displacement components 5 and 6 , per the example problem of Fig. 5

Clearly deformation $e_{k}$ is identified with primary loads $P_{\rho}$ on the truss. Similarly, adjoint deformation $\hat{e}_{k}$ is identified with (adjoint) loads $Q_{\rho}\left(\operatorname{sgn} u_{\rho}\right)$ via $\left(\tilde{e}_{k}=\Lambda \hat{e}_{k}\right.$ is introduced for simplicity)

$Q_{\rho}\left(\operatorname{sgn} u_{\rho}\right)-\sum_{k} B_{k \rho} \frac{S_{k} \tilde{e}_{k}}{\ell_{\ell}}=0$

The second and third constraints of [Q] together with (5) and (6) summarize the model of elastostatics for the truss structure, i.e. the requirements for equilibrium, compatibility, and (linear) constitutive relation are incorporated therein. The adjoint problem is relevant only in the interpretation for design, which is to be considered presently. Also, it is in the perspective of the larger problem that the characterization in extremum problem form has paricular significance.

The requirement for stationarity in [Q] with respect to the $k$-th element of the design variable is reflected in the optimality condition

$\varepsilon_{k} \tilde{\varepsilon}_{k}=\omega_{k} \Gamma-\lambda_{k}, \quad k=1,2, \ldots, M$.

Here the product of strains $\varepsilon_{k}=e_{k} / \ell_{k}$ and $\tilde{\varepsilon}_{k}=\tilde{e}_{k} / \ell_{k}$ measures unit mutual energy. The interpretation of (7) follows next, based on the KKT conditions associated with the lower bound constraint on $S_{k}$. According to the complementarity relation $\lambda_{k}\left(\underline{S}_{k}-S_{k}\right)$ for this constraint, $\left(\underline{S}_{k}-S_{k}\right)<$ $0 \rightarrow \lambda_{k}=0$. Identifying elements for which this condition is satisfied by the index set $\mathrm{I}_{D}$, from (7) $\varepsilon_{k} \tilde{\varepsilon}_{k}=\omega_{k} \Gamma, \quad k \in \mathrm{I}_{D}$,

$I_{D}$ identifies the design subset of truss members, and the result indicates that the unit mutual energy for elements in this set has value proportional to the respective unit relative cost index. On the other hand, $\lambda_{k}>0 \rightarrow S_{k}-\underline{S}=0$ and so we write

$\varepsilon_{k} \tilde{\varepsilon}_{k}<\omega_{k} \Gamma, \quad k \in \underline{\mathrm{I}}$,

where I stands for the set of element indicies identified with these conditions. Since by the KKT conditions $\lambda_{k} \geq 0$, the only remaining possibility is $\lambda_{k}=0$ and $S_{k}-\underline{S}=0$, say for elements $k \in \mathbf{I}_{a}$. Thus the interpretation of (7) is complete, with closure $\{k ; k=1,2, \ldots, M\}=\mathbf{I}_{D} \cup \underline{\mathrm{I}} \cup \mathrm{I}_{o}$. Note that in the more common "minimum compliance" problem where the objective is given by the inner product of load $P$ and displacement $u$, and where $\varpi_{k} \equiv 1$, (7) reduces to the requirement

$\left(\varepsilon_{k}\right)^{2}=\Gamma-\lambda_{k}, \quad k=1,2, \ldots, M$,

which expresses the familiar result that, for the optimal truss, unit strain energy has uniform value over elements in the "design set", and is bounded by this value for all others.

Given that the prescribed ground structure provides adequately for support of the designated loads, as implied in the definition of ground structure given above, presence of constraints $\left(\underline{S}_{k}-S_{k}\right) \leq 0$ assures that solution design for any cost distribution admissible within problem statement [Q] is a 
viable structure. Existence of solutions to [Q] can be argued simply through consideration of the inverse problem, i.e. we ask < given a design within the set of admissible designs, does there exist a unit relative cost field $\varpi_{j}$ such that the design is optimal? $>$. Based on determinacy of the system of necessary conditions plus constraints, as argued above, the answer is affirmative. Indeed, once primary and adjoint state variables are obtained for a given design, and noting uniqueness of the solution for these problems in elastostatics, unit cost factors $\omega_{k}$ (and multipliers $\lambda_{k}$ as well) may be calculated directly from (7) (and the remainder of the system). Since the argument applies for all admissible structures, completeness of the mapping between elements of the set of unit relative cost distributions and the set of structural designs is established as well.

One of the conveniences of the particular formulation [Q] for linear elastostatics is that this form is extended easily to model finite deformation structural mechanics. The nonlinear form results simply by replacing the linear deformation kinematics relations of the second and third constraints in [Q] by their more general form. If these relations are represented as

$\hat{e}_{j}-f\left(\nu_{\gamma}\right)=0, \quad e_{j}-f\left(u_{\gamma}\right)=0$,

then, as an example, the exact accounting of change of geometry has functions $f_{i}$ appear in form to reflect the Pythagorean theorem. Once the constraints are modified appropriately, all other aspects of the extended model for elastostatics and design follow automatically.

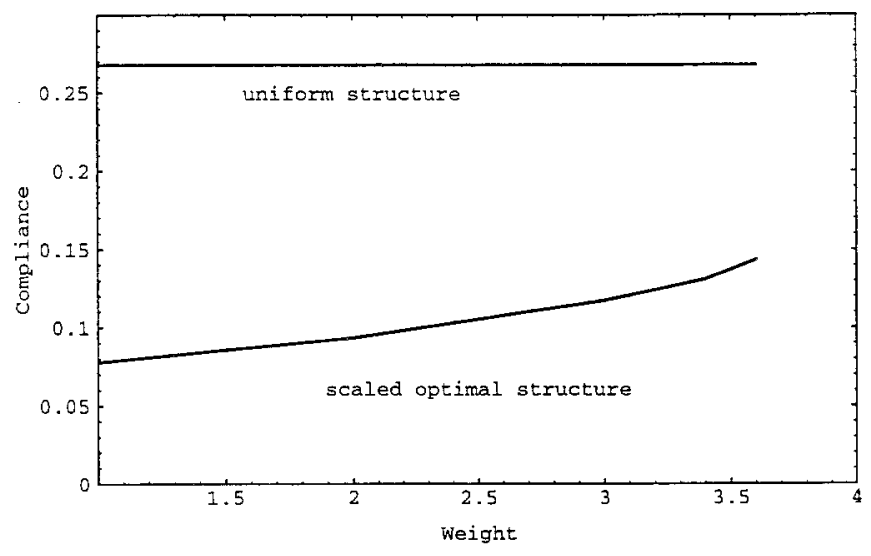

Fig. 8. Compliance versus weight factor for the example of Fig. 7

\section{Computational modelling and results}

All computational results presented here were obtained by use of an off-the-shelf program (Matlab optimization toolbox) that implements an SQP method for general constrained minimization problems. Relative effectiveness of different methods for computational treatment has not been studied. For a consideration of optimality criterion techniques, for example, see Rozvany and Zhou (1991). Most of the examples were submitted to the program in a form that parallels formulation [Q], the only exception being the introduction of means to maintain proper numerical conditioning by periodic scaling (the procedure used for scaling is routine, and this aspect of the computational model is not discussed here). Again, input data that serves to define the "ground structure" for a given problem has the form of coordinates for support nodes and interior nodes, and a connectivity matrix to designate which nodes are connected by a member. In the examples to be described below, each interior node is connected to every other node, i.e. the ground structure is complete.

Three example truss geometries are treated, all in 2D; their ground structrues are shown in Fig. 1. In the first example (Fig. 1a), torque load is to be transmitted between the outer and inner squares, and the design sought is simply the minimum compliance structure. Among results shown in Fig. 2, sketch (a) represents the solution obtained some time ago (Rossow and Taylor 1977) for the case of a uniformly weighted unit cost. Sketches (b)-(f) show various optimal designs' where a subset of elements (indicated as dashed lines) from the ground structure is given a heavily weighted unit cost, the remaining ones being held at uniform cost. The relative thicknesses of line segments representing members correspond to the relative stiffnesses of the members. This collection of predictions of optimal truss designs simply confirms the capability to solve the design problem for arbitrarily designated weight factors on unit cost, and they serve to exemplify the effect on design of emphasizing local cost over various parts of the ground structure.

Results are presented next (Fig. 3) for a nine-bar truss, first for uniform relative cost in sketch $3 c$, and then in the sketches $3 \mathrm{~d}-3 \mathrm{f}$ for increasing weight on the cost of members 1 and 2. An evolution of the optimal design with increasing weight factor applied to these two elements is depicted in Fig. 4. As an example of general compliance design, the same nine-bar truss is to be designed to minimize a weighted measure of displacement components 5 and 6 (these displacements are identified in Fig. 5a; the dashed lines with arrows shown there correspond to adjoint loads). The design result in Fig. 5b is optimal for uniform relative weight on nodal displacements, while sketches $5 \mathrm{c}$ and $5 \mathrm{~d}$ show optimal designs for specific higher weight factors for the designated displacement components 5 and 6 . The evolution of optimal designs under increasing weight on unit cost is given in Fig. 6 for this example problem.

Solutions for the final example problem proceed from the starting (ground) structure shown in Fig. $7 \mathrm{a}$, and the result in Fig. $7 \mathrm{c}$ is the optimal design for uniform unit cost on all members shown there. The remaining Figs. $7 \mathrm{~d}-\mathrm{f}$ represent a progression of solutions toward an end result that is to accommodate an obstacle in the central square of the layout, i.e. to provide a hole there in the final design. This is done simply by placing increasing values on the unit weight factors for all members passing through that region within the ground structure; these members are indicated in Fig. $7 \mathrm{~b}$ by dashed lines. Note that the lower bound value in the constraint on member stiffness is set to be relatively small, and that all members having stiffness at the lower bound are left off the diagrams. The curves of Fig. 8 provide information on the relative efficiency of the optimal designs, scaled for the purpose of comparison, relative to that of a structure with uniform members. Accordingly, the design of Fig. $7 \mathrm{c}$ is more than three times stiffer than the uniform truss. Almost onehalf of this gain is lost in the process to meet the requirement 


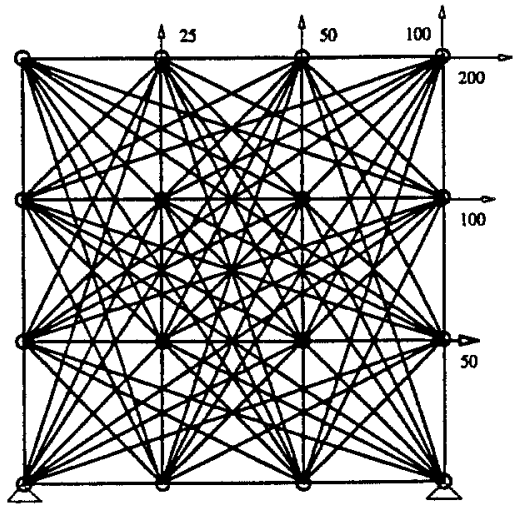

(a)

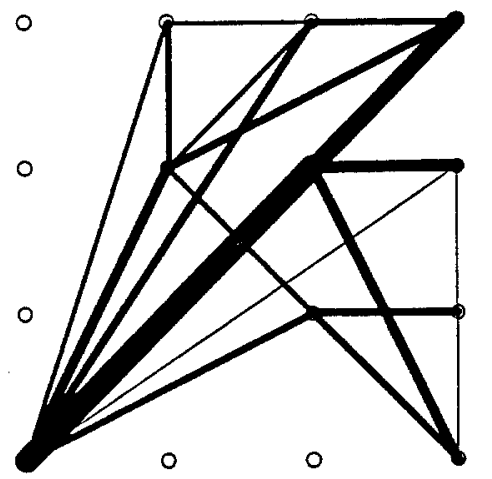

(c)

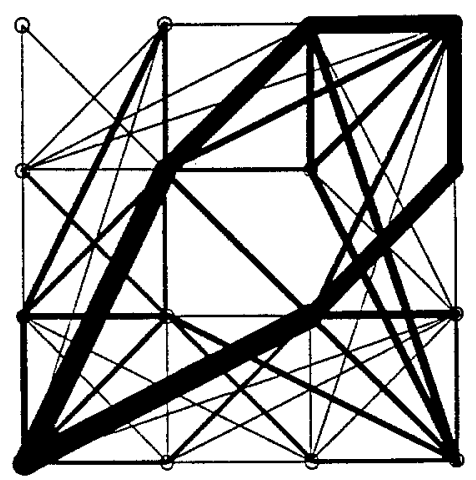

(e)

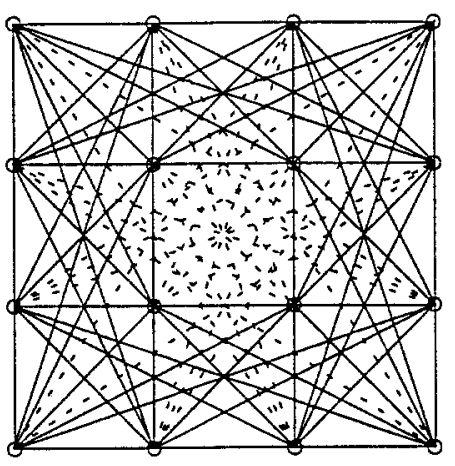

(b)

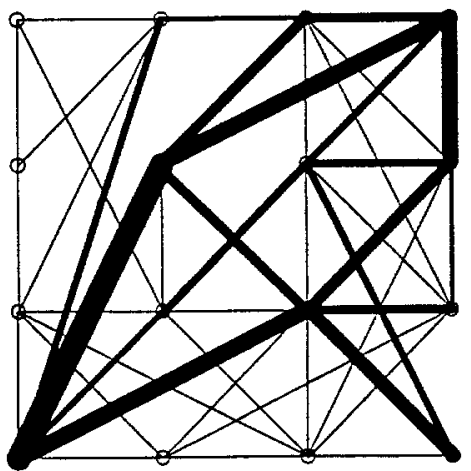

(d)

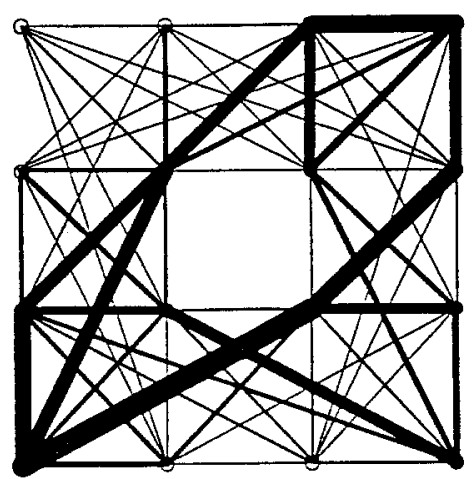

(f)

Fig. 7. Minimum compliance designs for the four-by-four truss example: (a) ground structure and loads, (b) elements with heavily weighted unit cost indicated by dashed lines, (c) results for uniformly weighted unit cost, (d)-(f) results for weight factor on costlier members at $2.0,3.15$ and 3.55 , respectively

for a hole in the final optimal design of Fig. 7f.

Two additional exercises were undertaken in connection with the latter example problem, namely to test certain procedures that would produce a cleaner final design, i.e. one with fewer members. In the first such procedure, all members of result Fig. $7 \mathrm{f}$ having stiffness below a chosen threshold level are given a relatively high unit cost factor. In this case, the optimization leads to the design of Fig. 9b. The second approach follows the procedure used in the original treatment of the problem, but starting from the much simpler ground structure shown in Fig. 9c; results in this case are given in Fig. 9d.

\section{Summary}

Formulation [Q] for truss design provides for the prediction of the optimal distribution of stiffness among the full array of members accommodated within a specified ground structure. Having the unit relative cost factors normalized provides for a comparison among designs for different unit cost distributions. Also, the problem [Q] represents a properly posed design problem for all admissible unit cost distributions, and thus the design problem model provides a mapping between the space of possible designs and the set of admissible unit cost distributions. On this basis it is reasonable to expect that there exists a solution to the inverse problem [this relates to statement (3) above (1)], i.e. the problem stated "what is 


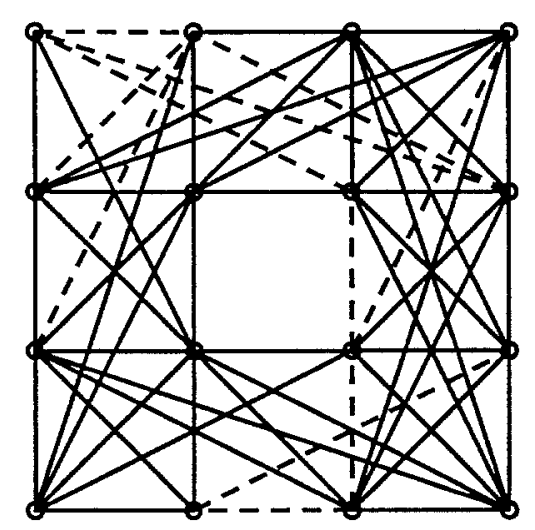

(a)

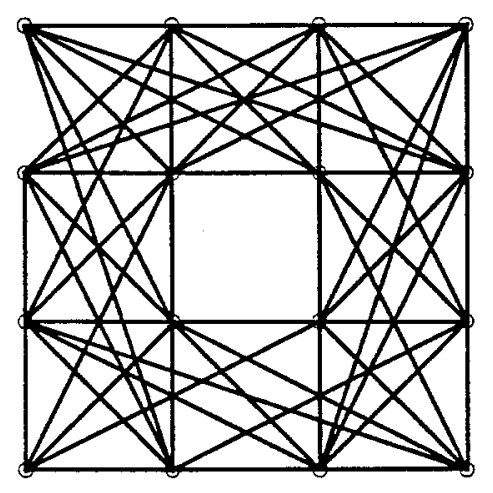

(c)



(b)

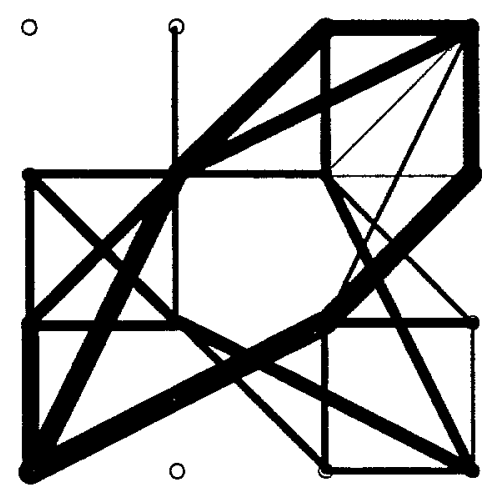

(d)

Fig. 9. Refined minimum compliance designs: (a) members shown as dashed lines given heavily weighted cost for the refinement, (b) resulting optimal truss starting from (a), (c) ground structure based on earlier results, (d) optimal truss design generated from the ground structure (c)

the unit relative cost distribution for which a given design is optimal?". The latter problem is, in fact, generally easier to solve than the forward one, and accordingly one might be encouraged to consider the design of algorithms for computational treatment that make use of the inverse problem form. The development of such computational means is to be undertaken in a later study.

The prediction of optimal topology design for continuum structures is in ways a more challenging problem than is the truss topology design treated here. This is manifest in part by the fact that existing techniques for the continuum problems, e.g. filtering schemes with penalization, perimeter methods, and so on are problematic still in their application. There is reason in heuristics to suppose that the generalized cost method described in this paper should be applicable to continuum design, and that, compared to familiar models, the application of such methods could be more successful. On the former point, one can visualize that the behaviour with respect to the design of a relatively fine, uniform-mesh truss with strictly local connectivity should simulate the behaviour of a continuum having similar support and load environment. The latter comment relates to the feature that has the form of the model in the generalized cost approach remain the same in the step by step procedure for computation (compared to what is done in familiar approaches where the objective itself is modified by the addition of penalty terms, or where the structure of the problem is otherwise altered within the computational procedures). Generalized cost models for the prediction of optimal topology for continuum structures, based in effect on a generalized cost extension of the formulation by Bendsøe et al. (1994) for the design of the material properties tensor, also are to be considered in a separate investigation.

\section{Acknowledgements}

The author is grateful for the help of Wendy Wilcox for her efforts toward generation of the computational example solutions. The work reported in this paper received support from the Ford Motor Company Project 95-106R, and from AFOSR contract \#f4962094-1-0442.

\section{References}

Bendsøe, M.P. 1995: Optimization of structural topology, shape and material. Berlin, Heidelberg, New York: Springer

Bendsøe, M.P.; Mota Soares, C.A. (eds.) 1993: Topology design of structures. Dordrecht: Kluwer 
Bendsøe, M.P.; Guedes, J.M.; Haber, R.B.; Pedersen, P.; Taylor, J.E. 1994: An analytical model to predict optimal material properties in the context of optimal structural design. J. Appl. Mech. 4, 930-937

Prager, W. 1977: Nearly optimal design of trusses. Comp. \& Struct. 8, 451-454

Rozvany, G.I.N. 1989: Structural design via optimality criteria. Dordrecht: Kluwer

Rozvany, G.I.N.; Zhou, M. 1991: Applications of the COC algorithm in layout optmization. In: Eschenauer, H.A.; Mattheck, C.; Olhoff, N. (eds.) Engineering optimization in design processes, pp. 59-70. Berlin, Heidelberg, New York: Springer

Rozvany, G.I.N.; Bendsøe, M.P.; Kirsch, U. 1995: Layout optimization of structures. Appl. Mech. Rev. 2, 41-119

Taylor, J.E. 1996: Generalized potential formulations for elastostatics of constitutively nonlinear structures under general load- ing. Int. J. Mech. Sci. 30, 537-548

Taylor, J.E.; Rossow, M.P. 1977: Optimal truss design based on an algorithm using optimality criteria. Int. J. Solids Struct. 13, 913-923

Taylor, J.E; Washabaugh, P.D. 1994: Analysis and design of trussed structures made of elastic/stiffening materials. Struct. Optim. 8, 1-8

Taylor, J.E.; Washabaugh, P.D. 1995: A generalized expression of cost for prediction of the optimal material properties tensor. In: Marques, M.D.P.M.; Rodrigues, J.F. (eds.) Trends in application of mathematics to mechanics. Essex: Longman

Washizu, K. 1982: Variational methods in elasticity and plasticity. Oxford: Pergamon Press

Zhou, M.; Rozvany, G.I.N 1992/1993: DCOC: an optimality criteria method for large systems. Part I: theory. Part II: alogrithm. Struct. Optim. 5, 12-25; 6, 250-262 